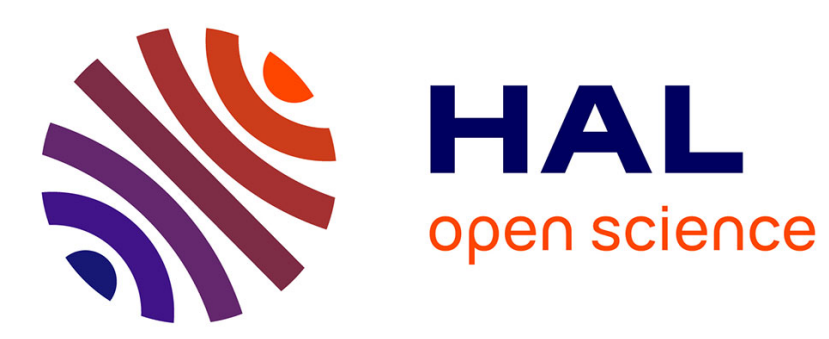

\title{
Une lecture régulationniste de la baisse de la part de la rémunération du travail dans la valeur ajoutée \\ Sandrine Michel
}

\section{To cite this version:}

Sandrine Michel. Une lecture régulationniste de la baisse de la part de la rémunération du travail dans la valeur ajoutée. Regards croisés sur l'économie, 2021, nº 27 (2), pp.48-57. 10.3917/rce.027.0048. hal-03370049

\section{HAL Id: hal-03370049 \\ https://hal.umontpellier.fr/hal-03370049}

Submitted on 7 Oct 2021

HAL is a multi-disciplinary open access archive for the deposit and dissemination of scientific research documents, whether they are published or not. The documents may come from teaching and research institutions in France or abroad, or from public or private research centers.
L'archive ouverte pluridisciplinaire HAL, est destinée au dépôt et à la diffusion de documents scientifiques de niveau recherche, publiés ou non, émanant des établissements d'enseignement et de recherche français ou étrangers, des laboratoires publics ou privés. 


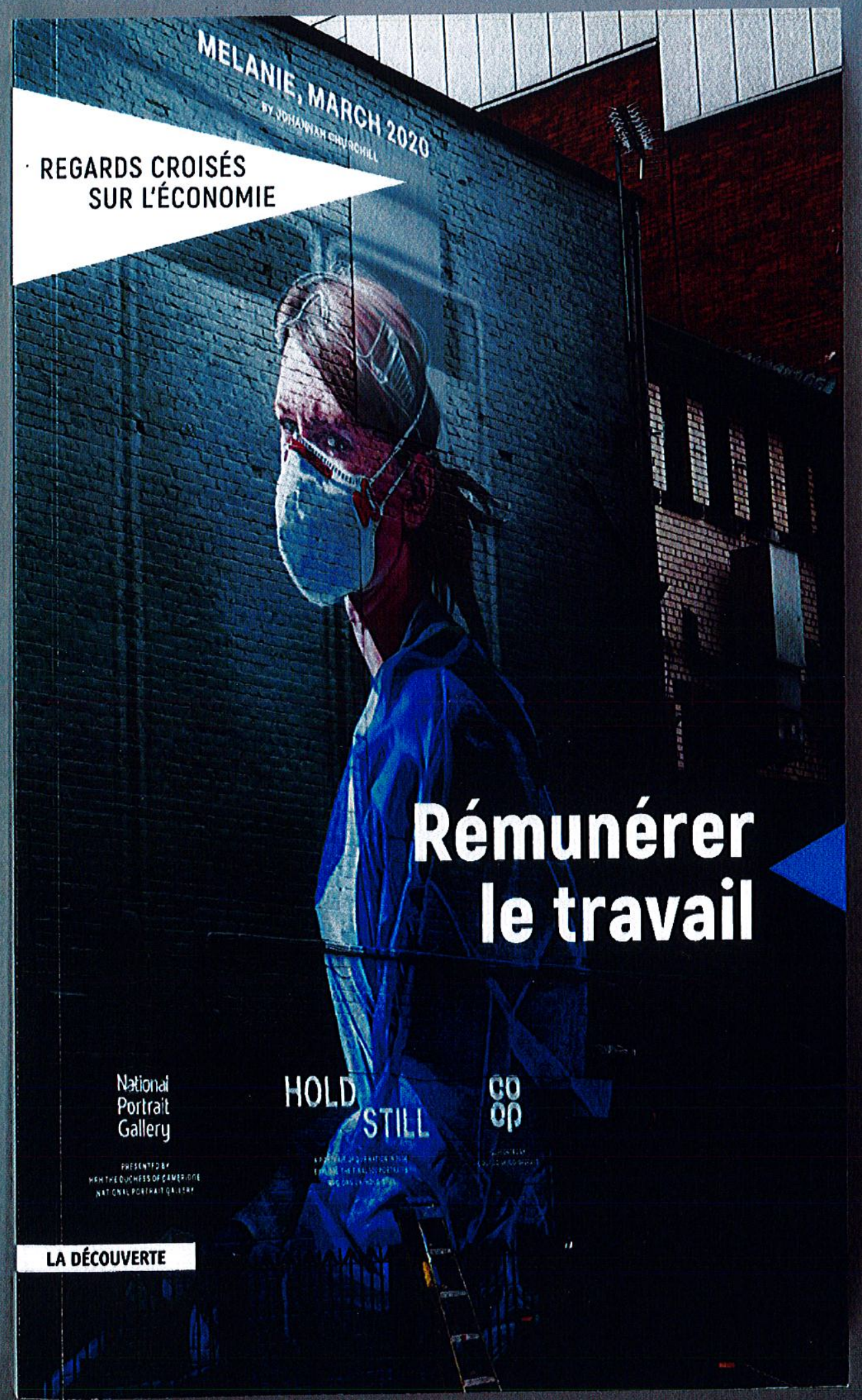




\title{
Une lecture régulationniste de la baisse de la part \\ de la rémunération du travail dans la valeur ajoutée
}

\author{
Sandrine MICHEL \\ ART-Dev, Université de Montpellier
}

L'analyse régulationniste du rapport salarial historicise le travail en tant que composante permanente des rapports de production et montre qu'il se transforme au gré des contraintes de l'accumulation et de la conflictualité sociale (Boyer, 2002). À chaque régime d'accumulation, défini comme un ensemble de régularités permettant d'assurer une évolution cohérente de l'accumulation du capital et de résoudre les distorsions auxquelles elle ne cesse de donner lieu, correspond une forme dominante de rapport salarial. La formation de la rémunération du travail est l'une de ces régularités.

Le rapport salarial fordiste (RSF), dernier rapport salarial stabilisé, s'est développé depuis 1945 dans les économies développées. Jusque dans les années 1970, il a assuré des débouchés à la production de masse via la progression ininterrompue du taux de salaire réel, part de la richesse revenant aux salariés, qui a assuré une augmentation de la demande globale (Aglietta 1976).

Avant 1945, la flexibilité des salaires et de l'emploi permettait d'absorber les difficultés conjoncturelles de l'accumulation tout en conduisant à son blocage structurel. Après 1945, le RSF généralise l'organisation collective des relations de travail à travers des institutions encadrantes telles que le salaire minimum, la négociation des salaires ou la norme d'emploi. Cette homogénéisation de la formation des salaires conduit à une baisse de la flexibilité du taux de salaire dans le fonctionnement des marchés du travail (Boyer, 1978).

Au début des années 1970, la crise de la rentabilité du capital pousse à des ajustements du régime d'accumulation qui expliquent la baisse durable de la part du travail dans la valeur ajoutée. Dans ce cadre, la remise en cause des modalités de l'institutionnalisation du RSF sera générale, à l'exception du salaire indirect. Quarante années de dégradation du RSF n'ont toutefois pas permis de faire émerger un nouveau rapport salarial.

\section{La rupture du compromis fordiste}

La formation du salaire fordiste se construit sur la base de l'équivalence entre le progrès de la productivité du travail et les gains salariaux. Cette équivalence a constitué la principale régularité du RSF et explique la croissance du salaire réel. Elle procède de processus de négociation entre les représentants des employeurs et des salariés dans les secteurs d'activités constituant le cœur du régime de croissance puis de leur généralisation. Sur ce point, les spécificités nationales sont nombreuses.

Part de la rémunération du travail dans la valeur ajoutée - Europe et Etats-Unis - 1950-2015 


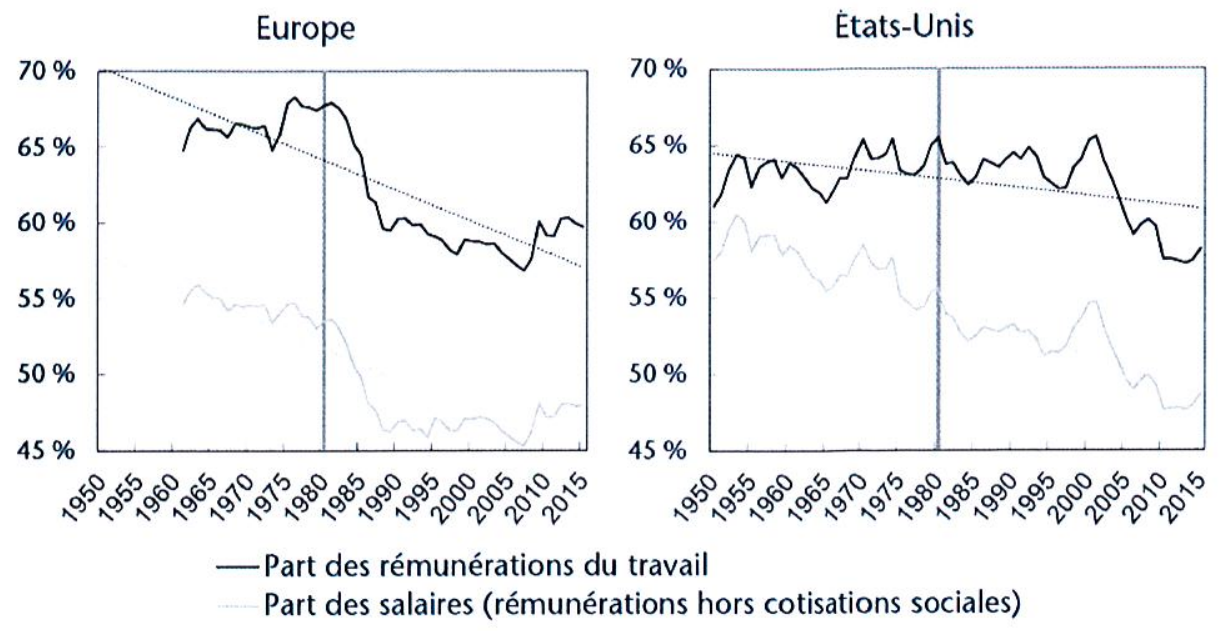

Source : Piton et Vatan (2018)

À partir des années 1970, la conjonction de difficultés de l'accumulation et du ralentissement des gains de productivité du travail crée des tensions dans la réalisation du compromis fordiste. À partir des années 1980, sa rupture s'observe partout. Elle généralise une progression ralentie du salaire réel qui reste, jusqu'à I'heure actuelle, très inférieure aux gains de productivité. Le transfert d'une partie de la richesse créée vers la rémunération du capital est ainsi enclenché.

Depuis les années 1950, l'indexation des salaires nominaux sur les prix avait permis de préserver le salaire réel des effets de l'inflation. En se basant sur l'inflation passée, elle contribuait à neutraliser la flexibilité du taux de salaire. Dans les années 1970, lorsque le taux de chômage commence à croître, l'indexation bascule sur la définition d'objectif d'inflation à atteindre. Elle flexibilise ainsi le marché du travail même si des mécanismes d'indemnisation du chômage contribuent à maintenir la demande globale. En France, l'indexation des salaires est abandonnée en 1983.

Le sous-emploi de masse a contribué à rétablir le chômage dans son rôle de modérateur des salaires en exerçant une pression durable à la baisse sur le taux de salaire. Du point de vue macroéconomique, la croissance de la productivité du travail redevient le seul motif de croissance du salaire. Or celle-ci est désormais très faible. Dans le même temps, l'ouverture des économies mettant face à face des niveaux de productivité et de salaires de différents pays conduit à une réorganisation massive des chaines de valeur et contribue à entretenir partout la pression sur les taux de salaire. La régulation concurrentielle conduit en effet à arbitrer en faveur des pays où rémunérer les gains de productivité est le moins coûteux, y compris en présence de progrès technique.

Les législations nationales, plus ou moins protectrices, extraient toujours des éléments de la norme d'emploi fordiste du champ concurrentiel. II en est ainsi du salaire minimum, étendu récemment à I'Allemagne ou en cours de généralisation aux Etats-Unis. La baisse du temps de travail a pu, comme en France en 2000 ou plus récemment dans certaines branches allemandes, conduire à réarmer ponctuellement une norme d'emploi. Cependant, le retour des mécanismes de marché dans la détermination de l'emploi et des salaires participe à la baisse de la part du travail dans la valeur ajoutée via une individualisation des relations d'emploi.

Ainsi, des négociations de l'emploi et des salaires se poursuivent mais au niveau des entreprises pour ajuster le travail en temps de crise (Perez et al., 2015). En France, la baisse du temps de travail a été financée par les salariés via les gains de productivité liés à une réorganisation du travail, négociée entreprise par entreprise (Billaudot, 2007). Ainsi, de nouvelles formes d'emploi se développent, positionnant parfois l'emploi à la frontière du salariat comme dans le cas du travail de plateforme. 
Dans ce contexte où chômage et hétérogénéité se combinent, la reprise de l'emploi n'est qu'exceptionnellement celle du salaire.

Après 1970, le progrès technique a contribué à une relance de l'accumulation sans modification du rapport capital /travail et donc de la répartition de la valeur ajoutée. En effet, les progrès ponctuels du taux de profit indiquent que le capital nouvellement accumulé a amplifié le ralentissement des gains de productivité du travail, privant la relance de l'accumulation de sa principale incitation. Dans un tel contexte, la financiarisation souligne une recherche alternative durable de gains pour du capital sans horizon de long terme : les activités pour soutenir un renouvellement de l'accumulation sont identifiées (recherche, éducation, santé, transition énergétique) mais leur potentiel de productivité reste flou.

\section{Une composante de la rémunération du travail fait de la résistance}

Dans ce contexte, l'évolution de la part du salaire indirect dans la répartition de la valeur ajoutée est atypique. Elle se maintient en Europe et augmente aux États-Unis (graphique 1).

Les premiers travaux sur le RSF ont mis en exergue la croissance des consommations collectives, certaines sous formes de salaire indirect comme la santé ou les retraites, d'autre sous forme de dépenses publiques comme l'éducation (Aglietta, 1976). L'analyse de ces consommations insiste sur la formation de compromis institutionnalisés anciens qui ont stabilisé la conflictualité sociale en architecturant les systèmes éducatifs ou les systèmes de protection sociale sur des normes publiques (Delorme \& André 1983).

L'analyse des rapports entre les dépenses sociales ou publiques à l'origine de ces consommations collectives et l'accumulation est graduelle. Leur essor contemporain est d'abord envisagé comme un produit joint de la croissance. La marchandisation de ces services collectifs, considérée comme une solution possible à leur forte croissance, n'advient pas (Billaudot et al., 1979). En conséquence, cette première approche considère qu'en phase critique, les dépenses attribuées au salaire indirect dégradent la rentabilité du capital et constituent, à ce titre, un obstacle à l'accumulation (Mazier et al., 1993). Leur maintien relatif lorsque la part de la rémunération du travail dans la valeur ajoutée baisse invite cependant à approfondir l'analyse.

D'autres travaux investissent la longe période pour comprendre comment dépenses sociales et d'éducation apparaissent et se structurent. Pour les pays développés, Lindert (2004) montre leur caractère cumulatif, leur faible sensibilité aux revers de la conjoncture et conclut à leur coût nul pour la croissance. En effet, en longue période, les coûts de ces dépenses et les gains de productivité qu'ils permettent sont équivalents.

En outre, les dépenses sociales et d'éducation tendent à devenir une régularité des différents rapports salariaux issus des phases dépressives et, en conséquence, des régimes d'accumulation successifs (Michel et Vallade, 2007). Si leur financement donne une occasion de dévaloriser du capital suraccumulé jusqu'en 1945, il suppose ensuite qu'une part de la valeur ajoutée leur soit dédiée (Michel et Vallade, 2010). En cela, ces dépenses sont intégrées au circuit du capital et sont parties prenantes de la dynamique de l'accumulation. Dans ce cas, pourquoi échappent-elles au processus de régulation dont a fait l'objet le salaire direct depuis les années 1980 ?

La première explication est dans la puissance des compromis institutionnels qui les fondent. Très audelà du travail, ces compromis ont ainsi acquis une dimension politique structurante (Amable et Palombarini, 2018). Cette dimension est indissociable de l'incapacité du marché à les organiser, du coût croissant de leur généralisation dans le temps et $d^{\prime}$ une architecture de financement publique et sociale. 
La seconde explication est dans les perturbations que ces dépenses introduisent dans la manière de faire croître la productivité du travail. Avec elles, l'efficacité des combinaisons productives dépend moins des économies de travail que de l'amélioration de sa qualité, à coûts croissants, sans qu'aucun mode de réglage de ces dépenses par rapport au régime d'accumulation n'ait émergé. Dans le même temps, pour le régime d'accumulation actuel, la qualité du travail constitue le seul gisement actif de gains de productivité du travail (Berlingieri et al., 2017). Elle est donc un support de relance des dynamiques concurrentielles pour l'accumulation incorporant du progrès technique. Mais elle est aussi dysfonctionnelle.

En effet, les compromis institutionnalisés à l'origine de ces dépenses n'ont jamais prévu leur limitation. Leur puissance explique la croissance auto-entretenue de l'éducation (ou de la santé) à l'échelle des générations (Michel 2002) mais aussi, désormais, à travers les temps sociaux à l'échelle du cycle de vie (Vallade 2002). Le marché demeurant impuissant à normaliser la croissance de ces dépenses, c'est la normalisation publique qui encadre leur progression.

A travers la définition d'objectifs sectoriels de maîtrise de ces dépenses, l'Etat joue un rôle central dans l'adaptation des appareils de production des consommations collectives. On observe ainsi un freinage de la dépense d'éducation non pas en volume mais rapporté aux effectifs, aboutissant à une dégradation rapide de la qualité de l'éducation. De même, la fiscalisation des dépenses pour la protection sociale fait circuler des objectifs de contrôle des dépenses tout en contribuant à abaisser la contribution des entreprises à leur financement. La maîtrise des dépenses publiques et sociales projette, pour les appareils de production de ces consommations collectives, l'existence de gains de productivité latents que des économies de travail permettraient de révéler. La crise sanitaire est un puissant révélateur du coût de la lutte contre ces coûts du travail (Boyer 2021) tandis que leur maintien dans la répartition de la valeur ajoutée souligne leur apport à l'accumulation.

\section{Conclusion}

La crise du régime d'accumulation a conduit à la baisse de la part du travail dans la valeur ajoutée. Le maintien de la part du salaire indirect généré par le RSF souligne l'émergence d'une contradiction dans le mode de croissance de la productivité, dont les échos s'accumulent dans des observations encore dispersées. La maîtrise des dépenses publiques s'apparente à une régulation de la croissance de ces dépenses. Mais le rationnement qui en découle se traduit aussi par de l'inefficacité et des inégalités sans pour autant apporter de réponse à la question d'un nouveau réglage du rapport capital/travail. Ce dernier est-il envisageable sans que la question de la transformation du travail et de sa rémunération ne revienne au centre du jeu?

Aglietta M. (1976) Régulation et crise du capitalisme, Paris : Calmann Levy.

Amable B. et Palombarini S. (2018) L'illusion du bloc bourgeois. Alliances sociales et avenir du modèle français, Paris : Raison d'agir.

Berlingieri G., Blanchenay P., Calligaris S. \& Criscuolo C. (2017), "Firm-level productivity differences: Insights from the OECD's Multiprod Project ", International Productivity Monitor, 32: 97-115.

Billaudot B., Granou A. \& Baron Y (1979) Croissance et crise, Paris : Maspéro.

Billaudot B. (2007) "Les 35 heures n'ont pas été une erreur... et les erreurs commises sont réparables ", Revue de la régulation, Juin http://journals.openedition.org/regulation/1311

Boyer R. (1978) « Les salaires en longue période », Economie et Statistique, 103 (1) : 27-57.

Boyer R. (2002) "Vingt ans de recherches sur le rapport salarial: un bilan succinct ", in : Boyer R., Saillard Y., Théorie de la régulation. L'Etat des savoirs, Paris : La Découverte : 106-114. 
Boyer R. (2021) Les capitalismes à l'épreuve de la pandémie, Paris : La Découverte.

Delorme A. \& André C. (1983) L'Etat et l'économie. Un essai d'explication de l'évolution des dépenses publiques en France (1870-1980), Paris : Seuil.

Lindert P. (2004) Growing Public: Social Spending and Economic Growth since the Eighteenth Century, Cambridge: Cambridge University Press.

Michel S. (2002) « Formation et croissance économique en longue période : vers une continuité des temps de formation sur le cycle de vie », Economies et Sociétés, F, 40 (3-4) : 533-566.

Michel S. \& Vallade D. (2007) "Une Analyse de long terme des dépenses sociales", Revue de la régulation, $\mathrm{n}^{\circ} 1$ | Juin, http://regulation.revues.org/index1507.html

Michel S. \& Vallade D. (2010) "Financement des dépenses sociales: les apports d'une analyse de long terme", Economie Appliquée, 63 (3) : 73-114.

Mazier J, Baslé M \& Vidal J.-F. (1993) Quand les crises durent, Paris : Economica.

Perez C., Thevenot N., Berta N., Brochard D., Delahaie N., Jallais S., Perraudin C., Sauviat C. \& Valentin J. (2015) « Modes d'ajustement par le travail en temps de crise : des relations professionnelles sous tension ", La Revue de I'IRES, 84 (1) : 61-90.

Piton S. \& Vatan A. (2018) "Le partage de la valeur ajoutée, un problème capital », in CEPII, L'Economie mondiale 2019, Paris : La Découverte : 67-81.

Vallade D. (2002) La dynamique du temps libre : un vecteur de recomposition des temps sociaux. Une analyse économique de longue période, 19ème et 20ème siècles, thèse, Université de Montpellier 1. 


\section{REGARDS CROISÉS SUR L'ÉCONOMIE Rémunérer le travail}

Reproduite en couverture de cet ouvrage, une fresque célèbre les travailleurs de "première ligne " de la crise sanitaire de 2020. Ailleurs, les pancartes de manifestants rappelaient: «Les applaudissements, ça ne paye pas les factures. » Ces frictions témoignent des natures multiples et parfois concurrentes des rémunérations, monétaires et symboliques, du travail.

Comment rémunérer le travail, et comment le rémunérer justement? Toute activité doit-elle faire l'objet d'une rémunération? Car rémunérer une activité en espèces sonnantes et trébuchantes l'arrache au domaine de la gratuité, tout en la signalant comme travail à part entière.

A travers le prisme des rémunérations se laisse saisir le profond renouvellement des mondes du travail en cours : croissance historique des indépendants, migrations, nouvelles technologies, nouveaux cadres juridiques. A l'heure où la crise sanitaire a renouvelé les débats sur la hiérarchie des salaires et l'utilité des métiers, Regards croisés sur l'économie a invité économistes, sociologues, historiens, politistes, philosophes et juristes à interroger les frontières du travail et les conditions de sa reconnaissance.
Sarah Abdelnour

Philippe Askenazy Christian Baudelot

Asma Benhenda

Sophie Bernard

Laurence Brunet

Claude Didry

Marc Fleurbaey

Jérôme Gautié

Olivier Godechot

Marlène Jouan

Sandrine Michel

Sidonie Naulin

Hélène Périvier

Hillel Rapoport

François Rivière

Pierre Rondeau

Luc Rouban

Maud Simonet

Grégory Verdugo regards croisés sur l'économi

vise à construire des ponts entre la recherche académique et le débat public. Clairs et didactiques, ses articles rendent compte des dernières avancées en sciences sociales, en faisant appel à des spécialistes scientifiquement reconnus.

www.rce-revue.com

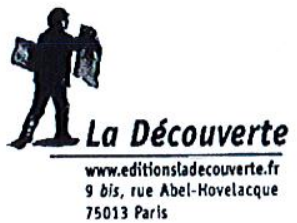

